\title{
QUALITY-BASED FUSION FOR MULTICHANNEL IRIS RECOGNITION
}

\author{
Mayank Vatsa ${ }^{1}$, Richa Singh ${ }^{1}$, Arun Ross ${ }^{2}$, and Afzel Noore ${ }^{2}$ \\ 1 - IIIT Delhi, India \\ \{mayank, rsingh\}@iiitd.ac.in \\ 2 - West Virginia University, USA \\ \{arun.ross, afzel.noore\}@mail.wvu.edu
}

\begin{abstract}
We propose a quality-based fusion scheme for improving the recognition accuracy using color iris images characterized by three spectral channels - Red, Green and Blue. In the proposed method, quality scores are employed to select two channels of a color iris image which are fused at the image level using a Redundant Discrete Wavelet Transform (RDWT). The fused image is then used in a score-level fusion framework along with the remaining channel to improve recognition accuracy. Experimental results on a heterogenous color iris database demonstrate the efficacy of the technique when compared against other score-level and image-level fusion methods. The proposed method can potentially benefit the use of color iris images in conjunction with their NIR counterparts.
\end{abstract}

Index Terms - Color iris recognition

\section{INTRODUCTION}

The human iris is a membrane composed of fibrovascular tissue or stroma that dilates or constricts the pupil thereby controlling the amount of light reaching the retina. The complex textural pattern on the anterior surface of the iris serves as a biometric cue for recognizing individuals. Iris recognition systems typically use near-infrared (NIR) sensors to image this complex pattern. This is because NIR illumination can penetrate the surface of the iris thereby revealing the intricate textural details of even dark-colored irides. The color of the iris, as revealed in the visible spectra (i.e., Red, Green and Blue channels, or RGB), is not used by most recognition systems. However, more recent research [1] has demonstrated the benefits of incorporating both color and texture information for iris matching. As can be seen in Fig. 1, the individual color channels can reveal complementary information, especially in the case of light-colored irides, which can be exploited by iris recognition systems.

With the advancement in sensor technology, color iris images are relatively easy to capture and therefore databases such as UBIRIS (v1 and v2), MILES, and UPOL are available for research. Boyce et al. [1] first explored the feasibility

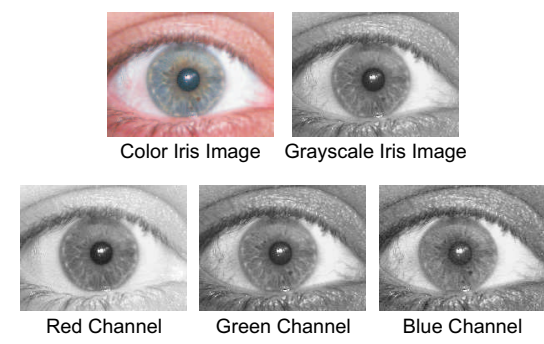

Fig. 1. A color iris image decomposed into three channels: Red (R), Green (G), Blue (B).
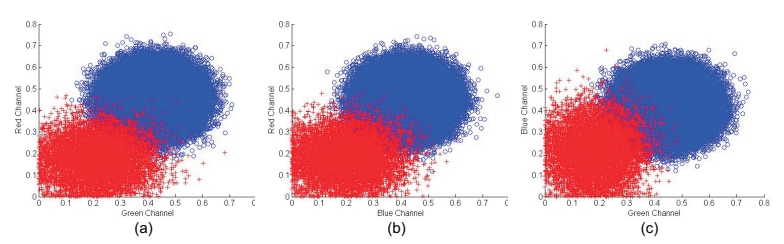

Fig. 2. Scatter plot of match scores between (a) red and green channels, (b) red and blue channels, and (3) green and blue channels. These scatter plots show that the match scores computed from different channels have limited correlation. Red points represent genuine scores and blue points represent impostor scores.

of using different color channels in conjunction with the NIR band to improve recognition accuracy. On a small dataset, the results indicated that the multichannel information has the potential to further enhance the iris recognition performance. Thereafter, Krichen et al. [2], Sun et al. [3], and Burge and Monaco [4] showed the usefulness of multichannel iris recognition.

In this paper, we present a fusion algorithm that uses multichannel color iris information to enhance recognition accuracy. The motivation behind the approach is based on observing the pair-wise correlation of match scores between the red, green and blue channels. Using the approach by Vatsa et al. [5] for iris segmentation, feature extraction and matching, the scatter plots of match scores between red-green, red-blue, and green-blue channels show (Fig. 2) that the scores are not 


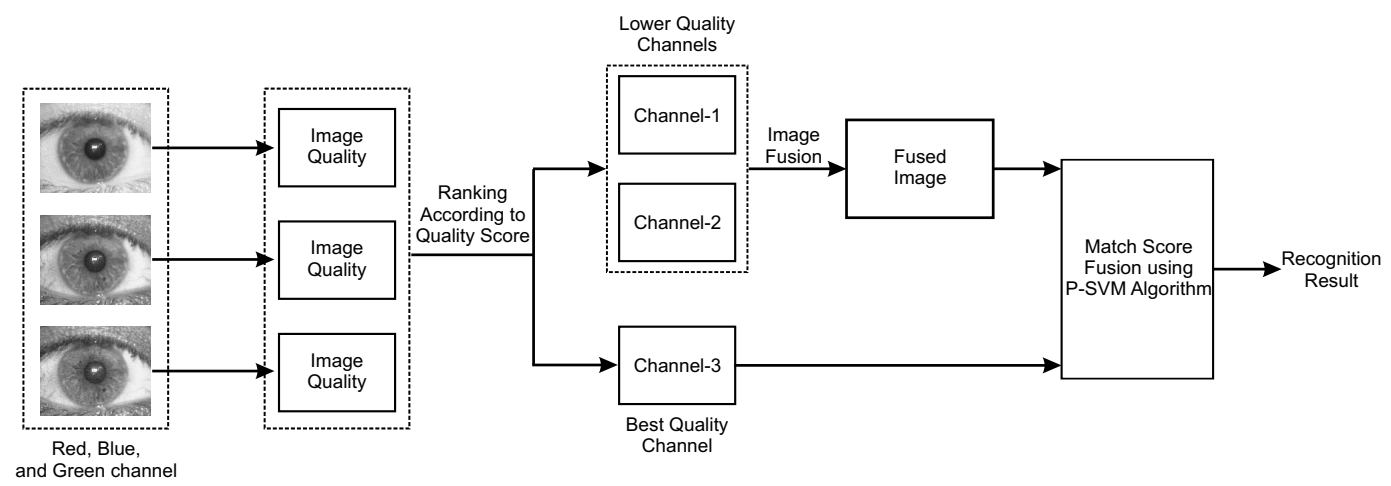

Fig. 3. Illustrating the steps involved in the proposed algorithm.

highly correlated. Further, when we compare the performance of individual color channels with the gray scale image (i.e., color iris images are converted into gray scale images), we observe that gray scale images provide better accuracy compared to individual channels (see Section 3). Since, we can view color-to-gray scale conversion as a simple image fusion technique, our analysis suggests that if we combine the multichannel information in a more systematic manner, the performance can be further improved. The proposed algorithm starts with computing the image quality of the probe color image based on the red, green and blue channels and ranks the individual channels based on quality. The two lowest quality channels are combined using the proposed image fusion algorithm and the resultant image is combined with the highest quality channel at the match score level. Fig. 3 illustrates the steps involved in the proposed algorithm.

\section{FUSION OF MULTICHANNEL IRIS IMAGES}

The proposed fusion algorithm that hierarchically performs image level fusion and match score level fusion is described in this section. The algorithm starts by segmenting iris images using the level set approach proposed by Vatsa et al. [5]. Segmented and unwrapped color iris images are then decomposed into red, green, and blue channels. A quality assessment algorithm [6], that encodes noise, blur, and off angle, is used to compute the image quality scores of the three channels independently. Based on the quality scores, we select the two lowest quality channels and use Redundant Discrete Wavelet Transform (RDWT) based image fusion to combine them. In the context of multichannel iris recognition, RDWT is preferred over DWT because it provides resilience to noise and is shift invariant. We select the lowest quality channels since RDWT can be used to glean useful information from these individual channels prior to fusing them. Thus, the noise components of these two channels are mitigated. Let $I_{c 1}$ and $I_{c 2}$ be the two channels. Three levels of RDWT decomposition is applied on both the channels to obtain the detail and approximation wavelet bands. Let $I_{c 1}^{a}, I_{c 1}^{v}, I_{c 1}^{d}$, and $I_{c 1}^{h}$ be the
RDWT subbands from $I_{c 1}$ channel. Similarly, let $I_{c 2}^{a}, I_{c 2}^{v}$, $I_{c 2}^{d}$, and $I_{c 2}^{h}$ be the corresponding RDWT subbands from $I_{c 2}$ channel. For the four subbands, each subband is divided into blocks of size $3 \times 3$ and the entropy of each block is calculated using Equation 1.

$$
e_{i}^{j k}=\ln \sqrt{\left(\frac{\mu_{i}^{j k}-\sum_{x, y=1}^{3,3} I_{i}^{j k}(x, y)}{\sigma_{i}^{j k}}\right) / m^{2}}
$$

where $j(=a, v, d, h)$ denotes the subbands, $m=3$ (size of each block), $k$ represents the block number, and $i(=c 1, c 2)$ is used to differentiate two channels $I_{c 1}$ and $I_{c 2} \cdot \mu_{i}^{j k}$ and $\sigma_{i}^{j k}$ are the mean and standard deviation of the RDWT coefficients of the $k^{t h}$ block of $j^{t h}$ subband respectively. Using the entropy values, the subbands for the fused image $I_{F}^{a}, I_{F}^{v}, I_{F}^{d}$, and $I_{F}^{h}$ are computed using Equation 2. In this image fusion scheme, more weight is given to the highest entropy image and the fused image block $I_{F}^{j k}$ is generated as:

$$
I_{F}^{j k}=\left\{\begin{array}{cc}
\omega_{1} I_{c 1}^{j k}+\omega_{2} I_{c 2}^{j k}, & \text { if } \quad\left(e_{c 1}^{j k}\right)>\left(e_{c 2}^{j k}\right) \\
\omega_{3} I_{c 1}^{j k}+\omega_{4} I_{c 2}^{j k}, & \text { otherwise }
\end{array}\right.
$$

Here, $\omega_{1}, \omega_{2}, \omega_{3}$, and $\omega_{4}$ are defined as,

$$
\begin{aligned}
& \omega_{1}=\frac{2 e_{c 1}^{j k}+e_{c 2}^{j k}}{e_{c 1}^{j k}+e_{c 2}^{j k}}, \quad \omega_{2}=\frac{e_{c 2}^{j k}}{e_{c 1}^{j k}+e_{c 2}^{j k}} \\
& \omega_{3}=\frac{e_{c 1}^{j k}}{e_{c 1}^{j k}+e_{c 2}^{j k}}, \quad \omega_{4}=\frac{e_{c 1}^{j k}+2 e_{c 2}^{j k}}{e_{c 1}^{j k}+e_{c 2}^{j k}}
\end{aligned}
$$

Finally, using Equation 4 inverse RDWT is applied on the fused subbands to generate the fused iris image, $I_{F}$. Fig. 4 shows an example where the blue and green channels of an iris image are fused.

$$
I_{F}=\operatorname{IRDWT}\left(I_{F}^{a}, I_{F}^{v}, I_{F}^{d}, I_{F}^{h}\right)
$$




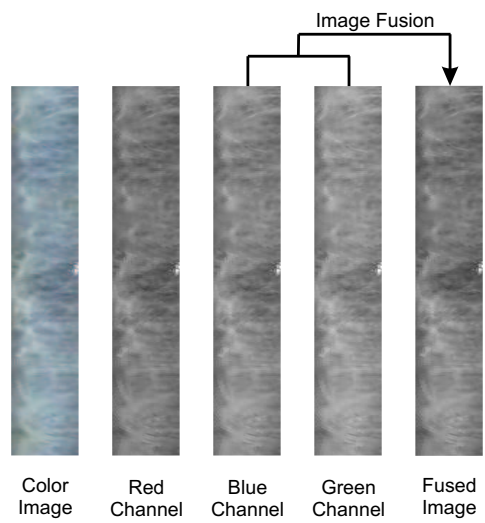

Fig. 4. Example illustrating the result of the proposed image fusion algorithm. Here, a normalized quality score of 0.94 is obtained for the red channel. The blue and green channels have quality scores of 0.85 and 0.81 , respectively. The fused image has a quality score of 0.92 .

In the next step, we individually extract and match iris features from the best quality channel and the fused image using the approach by Vatsa et al. [5]. Once the scores pertaining to the good quality channel and the fused image are obtained, we perform match score fusion using probabilistic support vector machine fusion (P-SVM) [7]. In this score fusion scheme, the likelihood ratio test statistic is integrated in a SVM framework. The score fusion can be denoted as

$$
M_{\text {fused }}=P S V M\left(M_{c 3}, M_{F}\right),
$$

where $M_{c 3}$ represents the match score obtained by matching the channel image with the highest quality, $M_{F}$ represents the match score obtained by matching the RDWT-fused image, $M_{\text {fused }}$ is the fused match score and $P S V M$ denotes P-SVM fusion.

\section{EXPERIMENTAL RESULTS}

The proposed algorithm is evaluated using a heterogenous color iris database. Description of the database and experimental protocol is explained in Section 3.1. Further, the product of likelihood ratio (PLR) based match score fusion [8] (i.e. fusion of match scores obtained from individual channels) and simple color-to-gray scale conversion (i.e. image fusion) are used for performance comparison.

\subsection{Database}

To evaluate the performance on a large number of iris classes, we combined multiple color iris databases. WVU multispectral iris database [1] contains multispectral iris images (RGB channels + NIR image) pertaining to 25 eyes with 5 images per eye. From this database, we used only the RGB channels and excluded the NIR component. Next, we used the
UPOL color iris database that contains 384 images pertaining to 128 eyes (i.e. 3 images per eye). Miles iris database ${ }^{1}$ is a high resolution color iris database. From this database we chose over 600 color images pertaining to 250 eyes. Finally, we combined color iris images from the UBIRIS v1 and UBIRIS v2 databases [9], [10]. Total number of images in the combined database is over 14,000 pertaining to 1,166 distinct eyes. From these images, 4200 images pertaining to 350 subjects ( $30 \%$ of complete images) are used for training and the remaining non-overlapping distinct eye classes are used as testing or gallery-probe set. This train-test partitioning is performed 10 times for cross validation. The experiments are performed for both verification (1:1 matching) and identification (1:N matching) scenarios. For verification, Receiver Operating Characteristics (ROC) curves are generated by computing the genuine accept rates (GAR) over the cross validation trials at different false accept rate (FAR). For identification, Cumulative Match Characteristic (CMC) plots are computed for identification accuracies ranging from rank 1 to 10 .

\subsection{Experimental Analysis}

Fig. 5 illustrates the performance of the proposed method along with the performance of individual channels, color-togray scale converted iris images, and PLR match score fusion. The key results and analysis of our experiments are summarized below.

- In general, red channel results in better performance compared to the other channels. This could be because the red channel shows high reflectance property and is very close to the NIR wavelength. It was also observed that the blue channel shows the least accuracy (as observed also by Boyce et al. [1]).

- Conversion from color to gray scale (simple image fusion) improves the recognition performance. Similarly, PLR match score fusion scheme shows better performance compared to individual channels. These results substantiate our hypothesis that combining multichannel iris information can improve performance.

- Quality scores obtained from fused images are, in general, 5-14\% higher than the two component images and very close to the highest quality (third) channel. However, there are cases when quality of all three channels are very high and the image fusion algorithm does not change the quality scores.

- The proposed hybrid fusion algorithm utilizes both image level fusion and match score fusion, thereby improving the performance significantly. t-test statistic suggests that at a $95 \%$ confidence interval, the proposed

\footnotetext{
${ }^{1}$ http://www.milesresearch.com/
} 

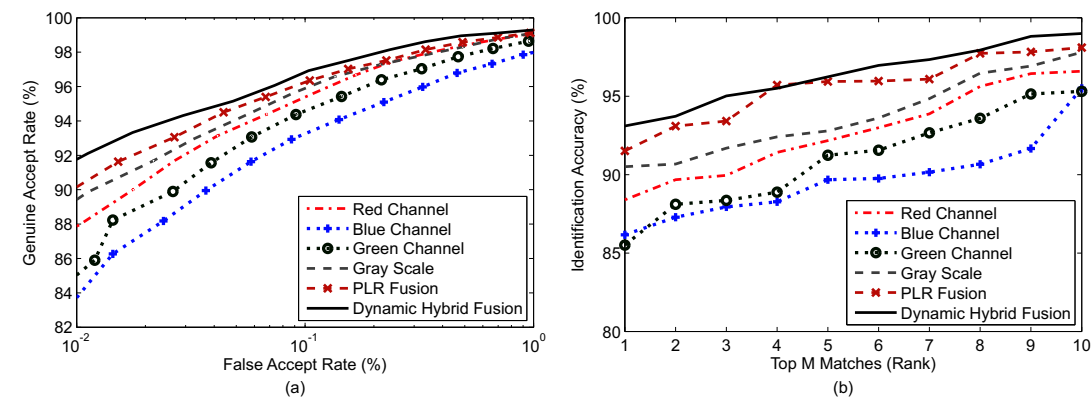

Fig. 5. Experimental results: (a) ROC plots and (b) CMC plots.

fusion algorithm is significantly different than PLR fusion and the color-to-gray scale conversion scheme. On combining the match scores of the fused image with that of the best quality channel, the verification accuracy at $0.01 \%$ FAR is $91.9 \%$ and rank-10 identification accuracy is $99.0 \%$.

- Best accuracy with the proposed fusion is lower than state-of-the-art results on other datasets. This is because UBIRIS $v 1$ and $v 2$ databases contain several nonideal images that reduce the performance significantly.

- On a $2 \mathrm{GHz}$ Pentium Duo Core processor with $4 \mathrm{~GB}$ RAM under $C$ programming environment, the proposed hybrid fusion algorithm requires around 1 second for segmentation, feature extraction, fusion and decision making. For identification, it requires less than 2 seconds to find Rank-10 matches. This computational time requirement is comparable to PLR match score fusion and slightly higher than color-to-gray scale conversion. Therefore, we can assert that the time requirement of the proposed approach is reasonable.

\section{SUMMARY}

A method to exploit the multiple spectral channels of an iris image has been proposed. The fusion method consolidates information at the image-level as well as the score-level in a hierarchical fashion. RDWT is used to fuse information at the image level by extracting the useful components from channels with the lowest quality. The resultant fused image is then used in a score-level fusion scheme along with the channel exhibiting the highest quality. The proposed method is observed to result in better performance than other score-level and image-level fusion methods. We are currently exploring methods to effectively match color iris images with their NIR counterparts.

\section{REFERENCES}

[1] C. Boyce, A. Ross, M. Monaco, L. Hornak, and X. Li, "Multispectral iris analysis: A preliminary study," in Proceedings of the 2006 Conference on Computer Vision and Pattern Recognition Workshop, 2006.

[2] E. Krichen, M. Chenafa, S. Garcia-Salicetti, and B. Dorizzi, "Color-based iris verification," in International Conference on Biometrics, 2007, pp. 997-1005.

[3] C. Sun, F. Melgani, C. Zhou, De Natale Francesco, L. Zhang, and X. Liu, "Semi-supervised learning based color iris recognition," International Conference on Natural Computation, vol. 4, pp. 242-249, 2008.

[4] M. J. Burge and M. K. Monaco, "Multispectral iris fusion for enhancement, interoperability, and cross wavelength matching," in Society of Photo-Optical Instrumentation Engineers (SPIE) Conference Series, 2009, vol. 7334, pp. 73341D$73341 \mathrm{D}-8$.

[5] M. Vatsa, R. Singh, and A. Noore, "Improving iris recognition performance using segmentation, quality enhancement, match score fusion, and indexing," IEEE Transactions on Systems, Man, and Cybernetics, Part-B, vol. 38, no. 4, pp. 1021-1035, 2008.

[6] N. D. Kalka, J. Zuo, N. A. Schmid, and B. Cukic, "Image quality assessment for iris biometric," in Biometric Technology for Human Identification III, 2006, pp. 61020D-1-62020D11.

[7] M. Vatsa, R. Singh, A. Ross, and A. Noore, "Likelihood ratio in a svm framework: Fusing linear and non-linear face classifiers," in IEEE CVPR Biometrics Workshop, 2008, pp. 1-6.

[8] K. Nandakumar, Y. Chen, S. C. Dass, and A. K. Jain, "Likelihood ratio based biometric score fusion," IEEE Transactions on Pattern Analysis and Machine Intelligence, vol. 30, no. 2, pp. 342-347, 2008.

[9] H. Proenca and L.A. Alexandre, "UBIRIS: A noisy iris image database," in International Conference on Image Analysis and Processing. 2005, vol. LNCS 3617, pp. 970-977, Springer.

[10] H. Proenca, S. Filipe, R. Santos, J. Oliveira, and L.A. Alexandre, "The UBIRIS.v2: A database of visible wavelength images captured on-the-move and at-a-distance," IEEE Transactions on Pattern Analysis and Machine Intelligence, vol. To appear. 